\title{
Evaluación de la Cromatografía lónica para Fomentar su Uso en la Investigación y Estudios de Posgrado en Ciencias del Agua
}

\author{
Eduardo Trujillo, Guadalupe Fonseca, Miriam A. García y Verónica Martínez \\ Universidad Autónoma del Estado de México, Facultad de Ingeniería, Cerro de Coatepec s/n, \\ C.U., 50130 Toluca, Edo. de México-México (e-mail: etrujillof@uaemex.mx, vmm@uaemex.mx)
}

\begin{abstract}
Resumen
Se evaluó la técnica de cromatografía iónica para fomentar su uso en la investigación y estudios de posgrado en ciencias del agua. Se analizaron muestras de agua natural con técnicas convencionales y HPLC y se encontró que la cromatografía iónica genera menos de la mitad del volumen de desechos con relación a las técnicas convencionales, desechos que son menos tóxicos y que están en concentraciones de $\mu \mathrm{g} / \mathrm{L}$. También, los costos se disminuyen 3.5 veces al utilizar HPLC, los tiempos promedio se reducen de 3 días a 4 horas, y los errores experimentales son menores. Los resultados encontrados permiten afirmar que la cromatografía iónica es una técnica analítica cualitativa y cuantitativa, rápida, económica, sensible y confiable y que puede ser realizada por estudiantes entrenados, no requiriendo de larga especialización.
\end{abstract}

Palabras clave: cromatografía iónica, actividades de investigación, ciencias del agua, enseñanza

\section{Evaluation of Ionic Chromatography to Motivate its Use in Research and Graduate Studies in Water Sciences}

\begin{abstract}
In this paper, ionic chromatography technique was evaluated to motivate its use in research and graduate studies in water sciences. Water samples were analyzed by both conventional techniques and HPLC and it was found that ionic chromatography generates less than a half of waste material than conventional techniques, wastes that are less toxic and with a level of concentration of $\mu \mathrm{g} / \mathrm{L}$ Also, when HPLC is used, the costs are reduced by a factor of 3.5 , the average time diminish from 3 days to 4 hours and the experimental errors are lower. The results show that ionic chromatography is a fast, economical, sensitive and reliable analytical technique that can be performed by trained students, not requiring highly specialized personnel.
\end{abstract}

Keywords: ionic chromotography, investigation activities, water sciences, teaching 


\section{INTRODUCCIÓN}

En los últimos 35 años, la técnica HPLC (High Performance Liquid Chromatography Cromatografía Líquida de Alta Resolución) se ha convertido en el método de separación analítico por excelencia, convirtiéndose en el tercer equipo estándar en los laboratorios analíticos, después de las balanzas y los potenciómetros (Kromidas, 2005). La cromatografía de líquidos se puede clasificar según la naturaleza de la fase estacionaria en cromatografía líquido-líquido, de adsorción, de exclusión molecular y de intercambio iónico o comúnmente llamada cromatografía iónica (Skoog y Haller, 2003). La constante búsqueda para la productividad creciente del laboratorio ha llevado al desarrollo de sistemas completamente automatizados, haciendo del HPLC un instrumento ideal para el análisis de una gama extensa de compuestos termolábiles no adecuados al análisis de la cromatografía de gases (Soniassy et al., 1994).

Actualmente, las nuevas técnicas de HPLC identifican, purifican y cuantifican, siendo de gran relevancia en casi todas las áreas de análisis instrumental por varias razones: a) el grado de automatización que ha alcanzado con las computadoras y el software, b) la capacidad y variedad de sus columnas, c) la diversidad de fases móviles a emplear, d) los detectores no destructivos, e) la inyección directa de mezclas complejas, f) su eficiencia y rapidez en el análisis de compuestos y/o iones, comparado con los métodos convencionales, y g) la detección de iones inorgánicos en bajas concentraciones (Skoog y West, 2004). Tradicionalmente, HPLC ha sido aplicado principalmente en la separación de vitaminas solubles en agua, esteroides y lípidos; vitaminas solubles en grasa, carbohidratos y ácidos; nucleómicos; proteómicos; para el monitoreo clínico y forense de drogas; para el monitoreo ambiental de aire y agua; y también para analizar contaminantes ambientales como plaguicidas y aromáticos polinucleares (McMaster, 2007). En general, HPLC es aplicada para determinar numerosos compuestos orgánicos en todo tipo de muestras, siendo particularmente apropiada para compuestos poco volátiles, de prioridad intermedia y alta y como técnica complementaria a la cromatografía de gases.

En cuanto al análisis de agua con HPLC, se han realizado trabajos desde distintos enfoques. Dawson et al. (2003), midieron las concentraciones de cloramina-T en aguas destinadas al cultivo de peces; Vichkovitten et al. (2007) han identificado bajas concentraciones de trihalosa en sedimentos de aguas costeras. Por otro lado, Amaro et al. (2005), estudiaron el contenido de $\mathrm{Cr}(\mathrm{VI})$ y $\mathrm{Cr}(\mathrm{III})$ en aguas de una industria de cromado antes y después de ser pasadas por un proceso de conversión y eliminación. Zhou et al. (1994), determinaron nitritos en agua, Moliner (2005) determinó amonio entre otros parámetros de calidad del agua, mientras que, Bruzzoniti et al. (2008) determinaron ácidos sulfónicos y alquilsulfatos en agua. En sistemas agua-suelo, Shimamoto y Takahashi (2008) se apoyaron en HPLC para determinar especies de yodo, mientras que Del Valle et al. (1996) detectaron CAAT (clorodiamina-s-triacina) en muestras de agua subterránea y suelos. Con relación a aguas naturales de ríos y líquidos cloacales, Sarno y Delfino (2001) determinaron compuestos fenólicos. Dionex Corporation ha identificado compuestos explosivos y sustancias relacionadas en agua para beber (Dionex, 2007); fenoles en agua mineral embotellada y para beber (Dionex, 2008); asimismo, ha realizado análisis de aniones inorgánicos en agua para beber (Dionex, 2003) y aguas ambientales (Dionex, 2001).

Muchos trabajos ambientales relacionados con el recurso hídrico usualmente están vinculadas a la determinación de iones individuales mediante métodos convencionales (volumétricos, turbidimétricos y espectrometría de absorción atómica). La cromatografía iónica cobra importancia en esta área ya que se puede identificar y cuantificar aniones y cationes en muy bajas concentraciones $(0.05 \mathrm{mg} / \mathrm{L}$ o menos, dependiendo del detector, fase móvil y definición de columna utilizados), sin emplear reactivos peligrosos. Dado que para el HPLC no existe un sistema de detección universal con alta sensibilidad como para cromatografía de gases, el detector a emplear depende principalmente de la naturaleza de la muestra y puede ser de ultravioleta, arreglo de diodos, fluorescencia o conductividad eléctrica (Skoog y Haller, 2003).

Organizaciones reguladoras como la USEPA, ASTM, AOAC, e ISO han avalado métodos basados en HPLC para el análisis de agua (Dionex, 2003). Los métodos 4110 A. de APHA-AWWA-WPCF y el 300.0 de la EPA proponen el uso de la cromatografía iónica como una técnica instrumental de medida secuencial y rápida para la determinación de diversos iones presentes en aguas superficiales, industriales, potables y subterráneas (APHA-AWWA-WPCF, 1992). 
Uno de los grandes retos que afrontan las instituciones de investigación y de estudios avanzados del ambiente, es obtener sus resultados basados en análisis químicos con calidad y rapidez, ya que ellos coadyuvan en la solución real y certera de los problemas ambientales.

En la Universidad Autónoma del Estado de México, las Facultades de Química e Ingeniería cuentan con equipos de HPLC. En la Facultad de Química, el equipo es utilizado principalmente para investigación en química orgánica, recientemente han comenzado a desarrollar trabajos de tipo ambiental. La Facultad de Ingeniería, a través del Centro Interamericano de Recursos del Agua ha utilizado el equipo para determinación de plaguicidas, compuestos organoclorados, e hidrocarburos aromáticos en agua superficial con vertidos industriales tratados.

El Centro Interamericano de Recursos del Agua oferta posgrados en Ciencias del Agua a nivel maestría y doctorado; la línea de investigación de Calidad del Agua engloba proyectos de investigación de profesores y proyectos-tesis de alumnos. A pesar de las ventajas de la técnica como tal, profesores-investigadores y alumnos no han podido acceder completamente a dichos beneficios, por aspectos como: a) baja confiabilidad de su aplicación en la determinación de iones en el agua al aplicar las técnicas de análisis convencionales aprobadas y recomendadas en las normas oficiales, b) desconocimiento de los costos de análisis por cromatografía iónica vs. técnicas convencionales y c) la falta de recursos humanos capacitados para la operación.

Muchos estudios que requieren la determinación de cationes y aniones en agua, pueden ser desarrollados de una manera fácil, rápida, económica, con mayor precisión y exactitud y sin generar grandes volúmenes de desechos tóxicos, si se utilizan técnicas de HPLC en lugar de las convencionales. Particularmente, las investigaciones de los alumnos de posgrado, suelen contar con presupuestos muy bajos, lo que les obliga a realizar menos muestreos y análisis, impidiéndoles desarrollar apropiadamente sus proyectos, situación que eventualmente repercute en los resultados globales obtenidos, en la adecuada solución de los problemas, y en su caso, en la limitada generación y aplicación de conocimiento.

Por lo tanto, el objetivo de este trabajo es realizar un comparativo de la aplicación de técnicas convencionales y de HPLC para la determinación de aniones y cationes en agua, valorando eficiencia, sensibilidad, confiabilidad, tiempo de análisis y costos, para aportar elementos que incentiven el uso de la cromatografía iónica, en la formación de estudiantes de posgrado e investigación en ciencias del agua.

\section{MATERIALES Y MÉTODOS}

\section{Preparación de muestras}

Se tomaron 20 muestras a lo largo de un curso de agua superficial alimentado por agua de manantiales y lluvia, para el cultivo de trucha, en aguas de este tipo es importante detectar los iones y trazas en bajas concentraciones. La toma y conservación de las muestras para ambas técnicas se realizó con base en lo establecido en la norma oficial mexicana. De acuerdo con el valor de $\mathrm{DBO}_{5}(<2 \mathrm{mg} / \mathrm{L})$ y el tipo de agua a estudiar, no fue necesario aplicar procesos para eliminar materia orgánica. Las muestras para cromatografía se filtraron 3 veces con membranas Millipore ${ }^{\circledR}$ de $0.45 \mu \mathrm{m}$ de tamaño de poro, con el fin de remover posibles partículas presentes, para su análisis se realizaron diluciones en función de la abundancia relativa de los constituyentes mayoritarios disueltos en agua subterránea (Chapman, 1992), las muestras diluidas se colocaron en viales, debidamente identificadas.

Se capacitó a un estudiante de Ingeniería Química en el manejo del equipo HPLC para que determinara catorce iones por cromatografía; se eligió aleatoriamente el $50 \%$ de ellos, para que el mismo estudiante los cuantificara por técnicas convencionales, para el otro $50 \%$ se consideraron los porcentajes de error obtenidos a través de cartas de control emitidas por el Laboratorio de Calidad del Agua del Centro Interamericano de Recursos del Agua. 


\section{Técnicas analíticas convencionales}

Se utilizaron técnicas analíticas convencionales de turbidimetría, volumetría y espectrometría de absorción atómica (Tabla 1), para la determinación de doce parámetros, con base en las normas oficiales mexicanas.

\section{Equipo de cromatografía}

Se utilizó un equipo de HPLC marca Thermofinnigan modelo Spectrasystem, que incluye los siguientes módulos: a) sistema de reservorio de fase móvil SCM 1000 con desgasificador; b) sistema de bombeo P2000; y c) sistema de inyección de muestra AS1000. Todos los módulos se controlan desde un sistema de comunicación ChromQuest. Se empleó un detector de conductividad, marca Alltech modelo 650. Se realizaron pruebas en diferentes flujos, iniciando por el recomendado por el sistema ChromQuest $(1.0 \mathrm{~mL} / \mathrm{min})$, variándose hasta que se obtuvo la mejor definición en el cromatograma, la cual fue de $0.8 \mathrm{~mL} / \mathrm{min}$ y un tiempo de estabilización de 20 minutos, siguiendo el método propuesto por Dionex $(2001,2003)$.

La calibración se llevó a cabo por medio de un estándar externo. La validación de los métodos con respecto a los límites de detección y de cuantificación, precisión y exactitud para cada método se realizó con base en lo establecido en los métodos estándar (APHA, AWWA-WPCF, 1992), así como en la respuesta de línea base. La precisión del método se evaluó en condiciones de repetibilidad y reproducibilidad, mientras que la exactitud del método se evaluó en términos de recuperación porcentual. La cuantificación se determinó por el área bajo la curva de cada pico, que es directamente proporcional a la concentración de cada ión, según el software del equipo.

Tabla 1: Técnicas analíticas convencionales

\begin{tabular}{|c|c|c|}
\hline Parámetro & Técnica/Norma Mexicana & Principio \\
\hline Alcalinidad & $\begin{array}{l}\text { Volumétrica } \\
\text { NMX-AA036-SCFT-2001 }\end{array}$ & $\begin{array}{l}\text { Determina la alcalinidad en el agua empleando como } \\
\text { disolución valorante una solución ácida de } \\
\text { concentración conocida. }\end{array}$ \\
\hline Cloruros & $\begin{array}{c}\text { Volumétrica } \\
\text { NMX-AA-073-SCFI-2001 }\end{array}$ & $\begin{array}{l}\text { A través de la formación de cloruro de plata utilizando } \\
\text { como indicador iones cromato. }\end{array}$ \\
\hline Dureza & $\begin{array}{c}\text { Volumétrica } \\
\text { NMX-AA.072-SCFI-2001 }\end{array}$ & $\begin{array}{l}\text { Considera la formación de complejos de EDTA con los } \\
\text { iones } \mathrm{Ca}^{2+} \mathrm{y} \mathrm{Mg}^{2+} \text {. }\end{array}$ \\
\hline N-amoniacal & $\begin{array}{l}\text { Volumétrica } \\
\text { NMX-AA-26-2001 }\end{array}$ & $\begin{array}{l}\text { El grupo amonio se alcaliniza con hidróxido de sodio, el } \\
\text { amoniaco liberado, se destila y se absorbe en una } \\
\text { disolución de ácido bórico, que posteriormente se titula. }\end{array}$ \\
\hline N-nitritos & $\begin{array}{l}\text { Colorimétrica } \\
\text { APHA-AWWA-WPCF } \\
(1992)\end{array}$ & $\begin{array}{l}\text { Se basa en la reducción del nitrato en nitrito en } \\
\text { presencia de cadmio, el nitrito se determina por } \\
\text { diazotización de la sulfanilamida para formar un azo. }\end{array}$ \\
\hline N-nitratos & $\begin{array}{c}\text { Colorimétrica } \\
\text { NMX-AA-079-SCFI-2001 }\end{array}$ & $\begin{array}{l}\text { El nitrito se determina por diazotización de la } \\
\text { sulfanilamida para formar un azo. }\end{array}$ \\
\hline P-fosfatos & $\begin{array}{c}\text { Colorimétrica } \\
\text { NMX-AA-029-SCFI-2001 }\end{array}$ & $\begin{array}{l}\text { Considera la reacción del fósforo con el ácido } \\
\text { molíbdico para formar ácido 12-molibdofosfórico el cual } \\
\text { es reducido por el cloruro de estaño. }\end{array}$ \\
\hline Sulfatos & $\begin{array}{c}\text { Turbidimétrica } \\
\text { NMX-AA-074-SCFI-2001 }\end{array}$ & El ión sulfato se precipita como sulfato de bario. \\
\hline $\begin{array}{l}\text { Sodio, potasio, } \\
\text { cinc, calcio, } \\
\text { magnesio }\end{array}$ & $\begin{array}{l}\text { Absorción atómica } \\
\text { NMX-AA-051-SCFI-2001 }\end{array}$ & $\begin{array}{l}\text { Contempla la generación de átomos en estado basal y } \\
\text { la medición de la cantidad de energía absorbida por } \\
\text { éstos. }\end{array}$ \\
\hline
\end{tabular}




\section{Determinación de aniones}

Se implementó el método met-anion-1 para identificar los aniones carbonato $\left(\mathrm{CO}_{3}{ }^{2-}\right)$, cloruros $\left(\mathrm{Cl}^{-}\right)$, fosfatos $\left(\mathrm{PO}_{4}{ }^{3-}\right)$, nitritos $\left(\mathrm{NO}_{2}{ }^{-}\right)$, nitratos $\left(\mathrm{NO}_{3}{ }^{-}\right)$y sulfatos $\left(\mathrm{SO}_{4}{ }^{2-}\right)$. Se utilizó una columna PXP$\mathrm{X} 100,100 \times 4.1 \mathrm{~mm}$, con fase móvil de ácido p-hidroxibenzoico 4.0mM, pH 8.9: metanol (97.5:2.5). Para establecer la curva de calibración se preparó una solución patrón de $50 \mathrm{mg} / \mathrm{L}$ para cada anión (a partir de un estándar de $1000 \mathrm{mg} / \mathrm{L}$ ) y posteriormente las soluciones estándar de $0.001 \mathrm{a}$ $15.0 \mathrm{mg} / \mathrm{L}$.

\section{Determinación de cationes}

Se implementó el método met-cation-3 para identificar los cationes amonio $\left(\mathrm{NH}_{4}^{+}\right)$, calcio $\left(\mathrm{Ca}^{2+}\right)$, cobalto $\left(\mathrm{Co}^{2+}\right)$, níquel $\left(\mathrm{Ni}^{2+}\right)$, magnesio $\left(\mathrm{Mg}^{2+}\right)$, potasio $\left(\mathrm{K}^{+}\right)$, sodio $\left(\mathrm{Na}^{+}\right)$y cinc $\left(\mathrm{Zn}^{2+}\right)$. Se utilizó una columna Universal Catión, $100 \times 4.6 \mathrm{~mm}$. Se probaron tres fases móviles diferentes, ácido metasulfónico $3 \mathrm{mM}$, ácido cítrico $5 \mathrm{mM}$ y ácido tartárico $2 \mathrm{mM} /$ ácido oxálico $1 \mathrm{mM}$, seleccionándose finalmente este último, debido a que presentó buena definición y se identificó una mayor cantidad de cationes. La calibración se llevó a cabo preparando una solución patrón de 50 $\mathrm{mg} / \mathrm{L}$ para cada catión (a partir de un estándar de $1000 \mathrm{mg} / \mathrm{L}$ ) y a partir de ésta se prepararon las soluciones estándar de 0.001 a $15.0 \mathrm{mg} / \mathrm{L}$.

\section{RESULTADOS Y DISCUSIÓN}

Mediante cromatografía iónica se identificaron y cuantificaron los iones mayoritarios (calcio, potasio, magnesio, sodio, carbonatos, cloruros y sulfatos), los secundarios (amonio, nitritos, nitratos y fosfatos) y los iones traza (cinc y cobalto), cuyas concentraciones se presentan en las Tablas 2 y 3 . En las figuras 1 y 2 se muestran dos de los cromatogramas generados por el equipo, donde se aprecian los picos de los iones determinados.

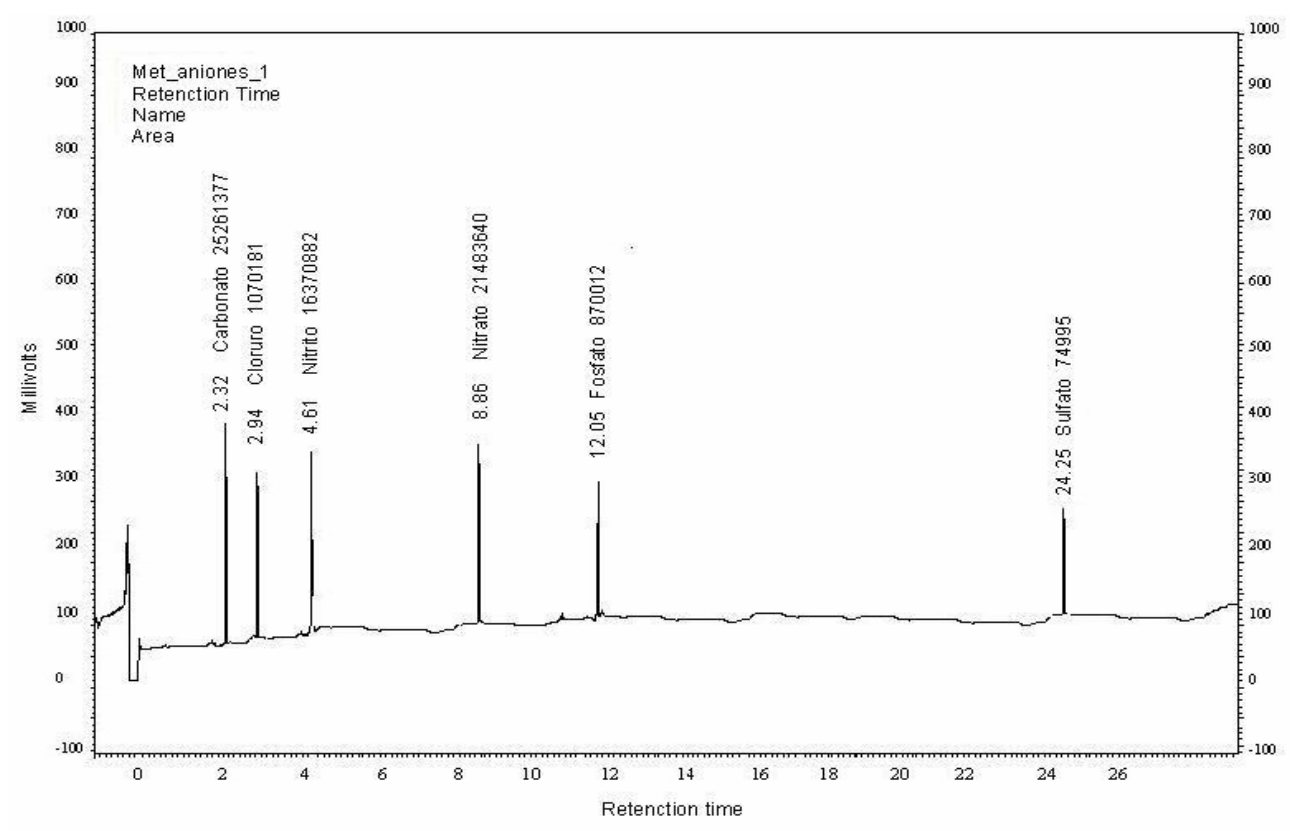

Fig. 1: Cromatograma de aniones

El tipo de agua estudiada es poco mineralizada y con presencia significativa de especies nitrogenadas, de ahí que únicamente se consideró la polaridad de la fase móvil como una de las condiciones para mejorar la eficiencia en la determinación de aniones, seleccionándose así la mezcla de ácido p-hidroxibenzoico:metanol sin tener la necesidad de utilizar un eluyente buffer, como lo recomienda Dionex (2003); para cationes es adecuada una fase móvil con una mezcla de ácidos tartárico y oxálico. Estas condiciones permitieron una buena sensibilidad y selectividad, así como una mayor precisión y exactitud. En la Tabla 2 se puede apreciar una mayor sensibilidad en la determinación de las concentraciones obtenidas por HPLC que por las técnicas convencionales. 


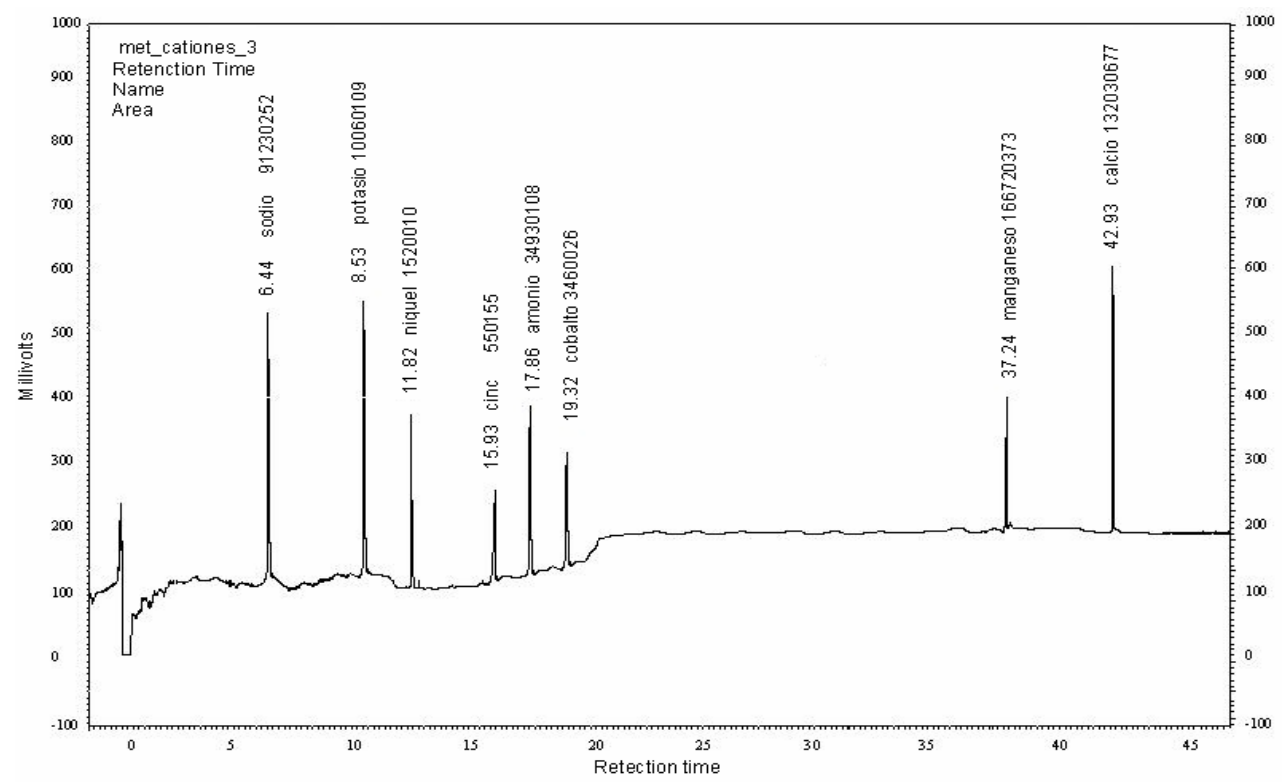

Fig. 2 Cromatograma de cationes

Tabla 2: Concentraciones obtenidas por métodos convencionales (C) y HPLC (H), en mg/L,

$\left(M^{\star}\right.$ : Número de Muestra; LD: Limite mínimo de detección del amonio $\left(10^{-4}\right)$, según APHA (1992))

\begin{tabular}{|c|c|c|c|c|c|c|c|c|c|c|c|c|c|c|}
\hline \multirow{3}{*}{$\mathrm{M}^{*}$} & \multicolumn{14}{|c|}{ lón } \\
\hline & \multicolumn{2}{|c|}{$\mathrm{NH}_{4}^{+}$} & \multicolumn{2}{|c|}{$\mathrm{Ca}^{2+}$} & \multicolumn{2}{|c|}{$\mathrm{CO}_{3}{ }^{2-}$} & \multicolumn{2}{|c|}{$\mathrm{Cl}^{-}$} & \multicolumn{2}{|c|}{$\mathrm{Mg}^{2+}$} & \multicolumn{2}{|c|}{$\mathrm{NO}_{3}^{-}$} & \multicolumn{2}{|c|}{$\mathrm{NO}_{2}$} \\
\hline & $\mathrm{H}$ & C & $\mathrm{H}$ & C & $\mathrm{H}$ & $C$ & $\mathrm{H}$ & C & $\mathrm{H}$ & C & $\mathrm{H}$ & $C$ & $\mathrm{H}$ & C \\
\hline 1 & 0.38 & $<1.0$ & 6.77 & 6.30 & 2.84 & 2.75 & 1.77 & 1.52 & 4.90 & 5.32 & 0.56 & 0.19 & 0.002 & 0.004 \\
\hline 2 & .55 & $<1.0$ & 7.74 & 8.48 & 1.30 & 1.52 & 1.64 & 1.52 & 4.76 & 4.79 & .10 & .16 & & \\
\hline 3 & .54 & $<1.0$ & 7.74 & 8.48 & 5.57 & 5.22 & 1.49 & 1.52 & 2.91 & 4.16 & $\overline{0.1}$ & .10 & .003 & 03 \\
\hline 4 & 0.05 & $<1.0$ & 9.62 & 9.89 & 3.45 & 4.17 & 1.50 & 1.52 & 3.82 & 3.82 & 0.13 & 0.12 & 0.006 & 0.004 \\
\hline 5 & $<\mathrm{LD}$ & $<1.0$ & 9.76 & 9.19 & 2.26 & 2.69 & 1.74 & 1.52 & 4.43 & 3.77 & 0.03 & 0.03 & 0.004 & 0.003 \\
\hline 6 & 0.85 & $<1.0$ & 10.5 & 9.89 & 2.24 & 2.22 & 3.45 & 3.79 & 3.92 & 3.90 & 0.0 & 0.03 & & \\
\hline 7 & 60 & $<1$. & & 14.1 & 2.48 & 2.84 & 3.17 & 3.7 & & 3. & & & & \\
\hline 8 & .01 & $<1.0$ & 11.2 & 8.48 & 2.23 & 2.69 & 3.23 & 3.79 & 4.24 & 3.94 & 0.2 & 0.02 & & \\
\hline 9 & 0.66 & $<1.0$ & 9.27 & 9.89 & 2.18 & 3.64 & 1.45 & 1.52 & 3.50 & 3.17 & 8.3 & 0.22 & 004 & 0.004 \\
\hline 10 & 0.67 & $<1.0$ & 9.53 & 9.18 & 2.24 & 2.60 & 1.58 & 1.52 & 3.35 & 2.91 & 0.36 & 0.23 & 0.004 & 0.004 \\
\hline 11 & $<\mathrm{LD}$ & $<1.0$ & 6.53 & 6.83 & 2.56 & 2.11 & 3.65 & 3.79 & 2.14 & 1.03 & 0.09 & 0.07 & .030 & 0.008 \\
\hline 12 & 0.17 & $<1.0$ & 6.36 & 6.24 & 3.39 & 3.17 & 3.46 & 3.79 & 2.09 & 1.53 & 0.02 & 0.03 & .030 & 0.020 \\
\hline 13 & & $<1.0$ & & 6.59 & 3.67 & 3.44 & 3.46 & 1.52 & & 1.65 & 0.0 & 0.09 & & \\
\hline 14 & $\mathrm{D}$ & $<1.0$ & & 5.28 & 2.32 & 2.11 & 2.86 & 1.52 & $\overline{93}$ & 1.18 & & 0.09 & & 0.0 \\
\hline 15 & $<\mathrm{LD}$ & $<1.0$ & 5.52 & 5.18 & 2.39 & 2.38 & 2.01 & 1.52 & 0.98 & 1.14 & 0.09 & 0.003 & 0.002 & 0.001 \\
\hline 16 & $<\mathrm{LD}$ & $<1.0$ & 4.98 & 4.94 & 3.54 & 3.45 & 2.56 & 1.52 & 1.47 & 1.65 & 0.01 & 0.01 & 0.003 & 0.009 \\
\hline 17 & $<\mathrm{LD}$ & $<1.0$ & 4.80 & 4.24 & 4.30 & 4.23 & 2.00 & 1.52 & 4.66 & 3.85 & 0.38 & 0.01 & 0.004 & 0.003 \\
\hline 18 & $<\mathrm{LD}$ & $<1.0$ & 5.06 & 5.65 & 3.30 & 3.17 & 2.81 & 3.03 & 1.91 & 2.04 & 0.03 & 0.02 & 0.052 & 0.015 \\
\hline 19 & $<\mathrm{LD}$ & $<1.0$ & 5.61 & 4.29 & 3.27 & 3.17 & 1.73 & 1.52 & 1.94 & 1.53 & 0.06 & 0.10 & 0.031 & 0.079 \\
\hline 20 & $<\mathrm{LD}$ & $<1.0$ & 6.79 & 5.00 & 3.49 & 3.45 & 1.86 & 1.52 & 1.93 & 1.20 & 0.08 & 0.10 & 0.004 & 0.057 \\
\hline
\end{tabular}

Las Tablas 4 y 5 muestran los porcentajes de error promedio para las dos técnicas utilizadas. En la tabla 5 se presentan los porcentajes de error derivados de técnicas convencionales establecidos por el Laboratorio de Calidad del Agua, según sus estándares de control. Se puede observar en la Tabla 4 que los errores obtenidos por el estudiante en las técnicas convencionales oscilan entre 6.4 y 18.5\%, mientras que por cromatografía iónica varían de 0.002 a 5.9\%.

En el caso de error de los parámetros por técnicas convencionales, en general, los valores del laboratorio (1.5-5.0\%) fueron menores que los del estudiante, tal y como se esperaba; con respecto a la cromatografía iónica (0.08-4.8\%) se observó un menor promedio de error (Tabla 5). Los iones níquel y cobalto son elementos que se encuentran a nivel traza en el agua, por lo que se requiere se evalúen bajo condiciones diferentes tanto de columna como de fase móvil (Dionex, 2003). 
Tabla 3: Concentraciones obtenidas por HPLC, en mg/L

\begin{tabular}{|c|c|c|c|c|c|c|c|}
\hline \multirow{2}{*}{$\begin{array}{c}\text { Núm. de } \\
\text { Muestra }\end{array}$} & \multicolumn{7}{|c|}{ lón } \\
\cline { 2 - 8 } & $\mathrm{PO}_{4}{ }^{3-}$ & $\mathrm{Ni}^{2+}$ & $\mathrm{K}^{+}$ & $\mathrm{Na}^{+}$ & $\mathrm{SO}_{4}^{2-}$ & $\mathrm{Zn}^{2+}$ & $\mathrm{Co}^{2+}$ \\
\hline 1 & 0.286 & 0.006 & 3.460 & 3.734 & 4.06 & 0.002 & $<0.001$ \\
\hline 2 & 0.005 & $<0.001$ & 2.532 & 3.463 & 5.68 & 0.001 & $<0.001$ \\
\hline 3 & $<0.001$ & 0.008 & 2.532 & 3.463 & 6.84 & 0.052 & 0.061 \\
\hline 4 & 0.501 & 0.017 & 3.349 & 4.629 & 8.54 & 0.032 & 0.014 \\
\hline 5 & 0.103 & 0.007 & 3.263 & 5.676 & 12.67 & 0.004 & 0.001 \\
\hline 6 & 0.120 & 0.001 & 4.036 & 5.491 & 10.57 & 0.015 & 0.026 \\
\hline 7 & 0.010 & 0.001 & 4.818 & 5.556 & 5.95 & 0.003 & 0.004 \\
\hline 8 & 0.016 & $<0.001$ & 3.635 & 6.058 & 5.71 & 0.005 & 0.001 \\
\hline 9 & $<0.001$ & 0.001 & 4.423 & 5.763 & 5.35 & 0.022 & $<0.001$ \\
\hline 10 & $<0.001$ & 0.011 & 5.525 & 5.761 & 5.79 & 0.095 & 0.050 \\
\hline 11 & 0.037 & 0.004 & 1.484 & 3.717 & 1.63 & 0.002 & 0.002 \\
\hline 12 & 0.179 & 0.004 & 1.566 & 3.697 & 1.75 & 0.001 & 0.003 \\
\hline 13 & $<0.001$ & 0.002 & 1.590 & 3.718 & 1.37 & 0.052 & 0.006 \\
\hline 14 & 0.057 & 0.022 & 1.637 & 3.794 & 5.23 & 0.032 & 0.013 \\
\hline 15 & $<0.001$ & 0.003 & 2.018 & 4.714 & 5.42 & 0.004 & 0.017 \\
\hline 16 & $<0.001$ & 0.022 & 0.957 & 3.521 & 6.34 & 0.015 & 0.021 \\
\hline 17 & $<0.001$ & 0.032 & 0.851 & 3.261 & 12.55 & 0.003 & 0.004 \\
\hline 18 & $<0.001$ & 0.036 & 1.224 & 3.537 & 11.89 & 0.005 & 0.028 \\
\hline 19 & $<0.001$ & 0.026 & 1.556 & 4.021 & 5.37 & 0.022 & 0.045 \\
\hline 20 & 0.031 & 0.019 & 1.555 & 4.31 & 3.07 & 0.095 & 0.014 \\
\hline
\end{tabular}

Tabla 4: Porcentajes de error registrados en las determinaciones del estudiante (El carbonato es calculado a partir de la alcalinidad)

\begin{tabular}{|l|c|c|}
\hline Parámetro & $\begin{array}{c}\text { \% de error. } \\
\text { Técnica Convencional }\end{array}$ & $\begin{array}{c}\text { \% de error. } \\
\text { Técnica HPLC }\end{array}$ \\
\hline Amonio & 11.55 & 3.9 \\
\hline Calcio & 18.48 & 5.9 \\
\hline Carbonato & 18.48 & 4 \\
\hline Cloruro & 16.70 & 4.2 \\
\hline Magnesio & 18.48 & 4.9 \\
\hline Nitratos & 6.44 & 5.64 \\
\hline Nitritos & 6.44 & 0.002 \\
\hline
\end{tabular}

Tabla 5: Porcentajes de error registrados en las determinaciones del estudiante - laboratorio (El cobalto y niquel no lo determina el laboratorio por técnicas convencionales)

\begin{tabular}{|l|c|c|}
\hline Parámetro & $\begin{array}{c}\text { \% de error. } \\
\text { Técnica Convencional }\end{array}$ & $\begin{array}{c}\text { \% de error. } \\
\text { Técnica HPLC }\end{array}$ \\
\hline Cobalto & - & 4.8 \\
\hline Fosfatos & 5.0 & 1.4 \\
\hline Níquel & - & 15.6 \\
\hline Potasio & 1.5 & 1.4 \\
\hline Sodio & 1.5 & 0.08 \\
\hline Sulfatos & 4.6 & 1.5 \\
\hline Cinc & 1.5 & 2.9 \\
\hline
\end{tabular}

En cuanto a los aspectos técnicos, analítico y económicos de HPLC con relación a los métodos convencionales, se identificaron las siguientes ventajas: a) el volumen de los residuos generados por HPLC (330 mL) por muestra con tres repeticiones, fue menos de la mitad del volumen generado por los métodos convencionales $(700 \mathrm{~mL})$, éstos últimos sin repeticiones; b) presenta un menor límite de detección y una mayor precisión; c) los residuos producidos por cromatografía iónica son de menor toxicidad y en concentraciones de $\mu \mathrm{g} / \mathrm{L}$, mientras que para los 
convencionales se incrementa la toxicidad y concentración a $\mathrm{mg} / \mathrm{L} ; \mathrm{d}$ ) el costo de análisis para los catorce iones, por HPLC ( $\$ 41$ USD) es 4.5 veces menor que por métodos convencionales (\$187 USD); e) la cromatografía iónica garantiza resultados con carácter de urgencia para los iones estudiados; el tiempo promedio para los análisis convencionales es de aproximadamente 3 días por muestra, mientras que por HPLC el tiempo promedio es de 4 horas, con tres repeticiones por muestra.

En cuanto a las desventajas observadas se encuentran: a) el costo inicial del equipo, b) el costo de contrato de mantenimiento (si se adquiere), c) el costo de los consumibles del equipo, d) el tiempo adicional para la estabilización del equipo y la columna, y d) la necesidad de contar con personal capacitado.

A través de la técnica de HPLC se obtienen resultados simultáneos de identificación y cuantificación de cationes y aniones presentes en el agua, esto se logra gracias al análisis concurrente de solutos en disolución acuosa utilizando columnas de alta definición, permitiendo el análisis secuencial de cationes mayoritarios (sodio, potasio, calcio y magnesio) y algunos trazas, como en este caso, cinc y cobalto. Aunque la columna utilizada en este estudio permitió determinar cinc y cobalto, la técnica recomendada es la espectroscopía de absorción atómica. Con respecto a las técnicas convencionales, éstas permiten determinar de forma individual la concentración de cada ión, lo que incrementa el tiempo de trabajo con relación a las técnicas de cromatografía.

La técnica instrumental (HPLC) se caracteriza por su simplicidad y tiempos cortos de análisis lo que ayuda a obtener una rápida caracterización de iones inorgánicos presentes en el agua (Hatsis y Lucy, 2003), contribuyendo al estudio de la calidad de aguas naturales (Whelan et al., 2004; Noij y Bobeldijk, 2003).

\section{CONCLUSIONES}

El análisis de los resultados, permite establecer las siguientes conclusiones:

1. La cromatografía iónica ha demostrado ser una técnica analítica de separación cualitativa y cuantitativa, rápida, económica, sensitiva y confiable, para la determinación de aniones, siendo una técnica alternativa a la espectroscopia de absorción atómica para el análisis de cationes presentes en agua natural.

2. El sistema HPLC básico, las columnas, solventes y mantenimiento, son costosos comparados con los equipos y materiales utilizados en las técnicas convencionales para la determinación de iones; sin embargo, por los beneficios que aporta la cromatografía, resulta ser una inversión conveniente.

3. Los desechos generados por HPLC son menos tóxicos y de menor volumen que los producidos por técnicas convencionales, lo que impacta en menor medida al medio ambiente.

4. Dado que gran parte del proceso está automatizado, los errores derivados de la intervención humana son mínimos cuando se emplea cromatografía iónica, además, la técnica puede ser accedida por personal afín a la química analítica, sin afectar significativamente la confiabilidad de los resultados. En estudios de posgrado, los estudiantes puede realizar sus propios análisis ante la falta de personal en los laboratorios.

5. HPLC puede contribuir a las investigaciones de profesores y estudiantes de posgrado, relacionadas con la calidad del agua, disminuyendo tiempos y optimizando presupuestos, sin menoscabar la confiabilidad de los resultados.

6. Los resultados encontrados en este estudio y las ventajas detectadas, sugieren la posibilidad de incorporar estas técnicas en las normas oficiales mexicanas. 


\section{AGRADECIMIENTOS}

A la Secretaría de Investigación y Estudios Avanzados de la Universidad Autónoma del Estado de México por el apoyo y financiamiento a este proyecto.

\section{REFERENCIAS}

Amaro, R. y otros cuatro autores; Seguimiento del Proceso de Conversión y Eliminación del $\operatorname{Cr}(\mathrm{VI})$ y $\mathrm{Cr}(\mathrm{III})$ en las Aguas de una Industrial de Cromado por HPLC-ICP-OES. Scientific Journal from the Experimental Faculty of Sciencies at La Universidad del Zulia: 13(1), 78-84 (2005).

APHA, AWWA, WPCF; Métodos Normalizados para el Análisis de Aguas Potables y Residuales. Díaz de Santos, S.A. $17^{\mathrm{a}}$ ed. pp 4.2 - 4.7, Madrid (España), 1992.

Bruzzoniti, M.C., R.M. De Carlo y C. Sarzanini; Determination of sulfonic acids and alkylsultates by ion chromatography in water. Talanta: 75(3), 734-739 (2008).

Chapman, D.; Water Quality Assessment, 5a ed., Edit. Chapman and Hall, pp 51-119, 371-461, (1992).

Dawson, V.K., J.R. Meinertz, L.J. Schmidt y W.H. Gingerich; A Simple Analytical Procedure to Replace HPLC for Monitoring Treatment Concentrations of Chloramine-T on Fish Culture Facilities. Aquaqulture: 217(1-4), 61-72 (2003).

Del Valle, L.P. y otros cuatro autores; Development of Improved Immunoassay and HPLC Methods for Analysis of Chlorodiamino-s-triazine in Environmental Samples, J. Agric. Food Chem.: 44, 945952 (1996).

Dionex, Corporation; Fast Analysis of Anions in Drinking Water by Ion Chromatography. Application Note 140, (en línea), 2001. http://www.dionex.com/en-us/webdocs/4093_140_V13.pdf. Acceso: 4 de mayo (2008).

Dionex, Corporation; Determination of Inorganic Anions in Environmental Waters Using a Hydroxide-Selective Column. Application Note 154, (en línea), 2003. http://www.dionex.com/enus/webdocs/4117_AN154_V19.pdf. Acceso: 6 de mayo (2008).

Dionex, Corporation; Determination of Explosive Compounds in Drinking Water Using ParallelHPLC with UVDetection. Application Note 189, (en línea), 2007. http://www.dionex.com/enus/webdocs/62167_AN189_hplc_explosivos_water_170ct07LPN1945.pdf. Acceso: 6 de mayo (2008).

Dionex, Corporation; Determination of Drinking and Bottled Mineral Waters Using Online Solid_Phase Extraction Followed by HPLC with UV Detection. Application Note 191, (en línea), 2008. http://www.dionex.com/enus/webdocs/61920_AN191_HPLC_Phenols_Water_30Apr08_LPN1949_02.pdf. Acceso: 4 de mayo (2008).

Hatsis, P. y C.A. Lucy; Improved Sensitivity and Characterization of High-speed ion Chromatography of Inorganic anions. Analytical Chemistry: 75(4), 995-1001 (2003).

Kromidas, S.; More Practical Problem Solving in HPLC, pp 269, WILEY-VCH Verlag GmBH \& Co. KGaA, Weinheim. Federal Republic of Germany (2005).

McMaster, M.C.; HPLC, a Practical User's Guide. $2^{\text {nd }}$ ed. Pp 159-166. John Wiley \& Sons, Inc. Hoboken, New Jersey (2007). 
Moliner, M.Y.; Aportaciones de la Química Analítica a la Resolución de Diversos Problemas Medioambientales. Tesis de Doctorado. Universitat de Valencia. Server de Publicacions, Valencia, España (2005).

NMX-AA-026-SCFI-2001; Secretaria de Economía. DGN. Análisis de Agua. Determinación de Nitrógeno Total Kjeldahl en Aguas Naturales, Residuales y Residuales Tratadas (2001)

NMX-AA-029-SCFI-2001; Secretaria de Economía. DGN. Análisis de Agua. Determinación de Fósforo Total en Aguas Naturales, Residuales y Residuales Tratadas (2001).

NMX-AA-036-SCFI-2001; Secretaria de Economía. DGN. Análisis de Agua. Determinación de Acidez y Alcalinidaden Aguas Naturales, Residuales y Residuales Tratadas (2001).

NMX-AA-051-SCFI-2001; Secretaria de Economía. DGN. Análisis de Agua. Determinación de Metales en Aguas Naturales, Residuales y Residuales Tratadas (2001).

NMX-AA-072-SCFI-2001; Secretaria de Economía. DGN. Análisis de Agua. Determinación de Dureza Total en Aguas Naturales, Residuales y Residuales Tratadas (2001).

NMX-AA-073-SCFI-2001; Secretaria de Economía. DGN. Análisis de Agua. Determinación de Cloruros en Aguas Naturales, Residuales y Residuales Tratadas (2001).

NMX-AA-074-SCFI-2001; Secretaria de Economía. DGN. Análisis de Agua. Determinación del lón Sulfato en Aguas Naturales, Residuales y Residuales Tratadas (2001).

NMX-AA-079-SCFI-2001; Secretaria de Economía. DGN. Análisis de Agua. Determinación de Nitratos en Aguas Naturales, Residuales y Residuales Tratadas (2001).

Noij, T.H.M. e I. Bobeldijk; Novel monitoring concepts to acquire new water quality knowledge. Water Sicience \&Technology: 47(2), 181-188 (2003).

Sarno, M. y M. Delfino; Fenoles y Derivados por HPLC-SPE. Ciencia y Tecnología, Comunicaciones Científicas y Tecnologicas 042 (en línea), 2001. http://www.unne.edu.ar/Web/cyt/2001/8-Exactas/E-Indice.htm. Acceso: 23 de Julio (2008).

Shimamoto, Y. e Y. Takahashi; lodine speciation in the soil-water systems using XANES an HPLCICP-MS. Geophysical Research Abstracts, 10(EGU2008-A-06989). (en línea), 2008. www.cosis.net/ abstracts/EGU2008/06989/EGU2008-A-06989.pdf?PHPSESSID=. Acceso: 11 de Agosto 2008.

Skoog, D. y D. West; Química Analítica. Mc. Graw Hill. $7^{a}$ ed. pp 703-715. México (2004).

Skoog, D. Y J. Haller; Análisis Instrumental. Mc. Graw Hill. 5ª ed. pp 652-671. México (2003).

Soniassy, B.; P. Sandra y C. Schlett; Water Análisis. Hewlett Packard. $1^{\text {a }}$ ed. Germany (1994).

Vichkovitten, T., M. Homer y M.S. Frederiksen; Spatial and Temporal Changes in Non-structural Carbohydrate Reserves in Eelgrass (Zostera marina L.) in Danish Coastal Waters. Botanica Marina: 50(2), 75-87 (2007).

Whelan, R. J.; T.E.Hannon, R.N. Zare y D.J. Rakestraw; Application of ion Chromotography to the Investigation of Real-world Samples. Journal of Chemichal Education: 81(9), 1299-1302 (2004).

Zhou, J.Y.; P. Prognon; C. Dauphin, y M. Hamon; HPLC Fluorescence Determination of Nitrites in Water Using Precolumn Derivatization with 4-methyl-7-aminocoumarin. Chromatographia: 36(1), 1612-1112 (1994). 